\title{
Gestational Weight Gain and Large for Gestational Age Neonates in a Predominantly Hispanic Population Community Hospital
}

\author{
Brittany N Robles*, Luz M Cardenas, Moez Sumar, Lucia Gonzalez, Lesley Hall, Giovanna Ramirez Barbieri, \\ Daniel Faustin and Ralph Ruggiero
}

OBGYN, Wyckoff Heights Medical Centre, USA

*Corresponding author: Brittany N Robles, Department of Obstetrics and Gynaecology and Reproductive Sciences, Wyckoff Heights Medical Centre, Brooklyn, NY, 11237 USA.
Received Date: April 20, 2020

Published Date: April 27, 2020

\begin{abstract}
To analyse changes in Gestational Weight Gain (GWG) in different Body Mass Index (BMI) categories at start of pregnancy and neonatal birth weight outcomes in a predominantly Hispanic population living in a low-income environment.

We conducted a cross sectional study of women with singleton gestation who delivered at Wyckoff Heights Medical Centre from January $1^{\text {st }}$ to December 31 $31^{\text {st }}$ 2017. BMI was categorized at first prenatal visit as normal weight (BMI= 18.5-24.9), overweight (BMI= 25-29.9) and obese (BMI >30). BMI was collected at 16-20, 24-28, and 36-38 weeks of gestation. Stratified by BMI, appropriate GWG were 25-35 pounds for normal weight (27.9\%), 15-25 pounds for overweight (34.5\%), and 11-20 pounds for obese (24.5\%). Neonatal birth weight was categorized by Duryea percentiles and gestational age in weeks. From 831 women, normal weight $(n=269)$, overweight $(n=263)$, and obese $(n=299)$ women were found. GWG was categorized as: inadequate, appropriate, or excessive based on the Institute of Medicine guidelines. The prevalence of excessive GWG was $23.1 \%$ for normal weight, $35.8 \%$ for overweight, and $37.5 \%$ for obese women. The prevalence of inadequate GWG was $48.9 \%$ for normal weight, $31.0 \%$ for overweight, and $40.6 \%$ for obese women. A significant association was found between obese women and $>90$ percentile neonatal birth weight (OR:2.3, 95\% CI: 1.15-4.97). Obese women were more likely to have excessive GWG which is associated with maternal and neonatal adverse outcomes such as NICU admissions, gestational diabetes, hypertensive disorders in pregnancy, delivery by caesarean section, large for gestational age, and shoulder dystocia.
\end{abstract}

Keywords: Gestational weight gain; Maternal obesity; Pregnancy weight gain; Pregnancy complications

\section{Introduction}

Gestational weight gain (GWG) is the amount of weight one gains throughout the pregnancy. This weight gain can be influenced by many factors including: age, socioeconomic factors, ethnicity, and maternal comorbidities, such as pre-gestational obesity [1-4]. Excessive gestational weight can lead to numerous adverse maternal and neonatal events such as the development of gestational diabetes, gestational hypertension, and preeclampsia, delivery via caesarean section, macrosomia, neonatal hypoglycaemia and shoulder dystocia. Due to the complications that can arise, recommendations were set forth by The Institute of Medicine (IOM) designating the appropriate amount of weight women should gain based on her pre-pregnancy BMI [5]. Although these guidelines were set forth just eleven years ago in 2009, they are debatable and do not take into consideration various important factors such as: weight gain among different ethnic groups, those of low socioeconomic status and/or those with a lower education level [5].

In the United States, more than $40 \%$ of pregnant women exceed the Institute of Medicines guidelines [6]. Excessive gestational weight gain varies by ethnicity and socioeconomic status with low income populations and non-whites being at the greatest risk [1].

Hispanics are the largest minority group living in the United States. They have the highest rates of inadequate and excessive GWG and are the group with the highest birth rate [6]. Hispanic women, specifically those from the Caribbean, experience the 
greatest health disparity. Furthermore, this subgroup of women has the highest prevalence of maternal comorbidities including obesity and type 2 diabetes mellitus and exhibit adverse outcomes associated with poor nutrition [6].

Women who are classified as severely obese at conception have an increased risk of infant mortality, stillbirth, congenital malformations, large for gestation infants, hypertensive disorders of pregnancy, gestational diabetes, prolonged second stage of delivery, delivery via caesarean section and maternal mortality than non-obese women [7].

In our predominantly Hispanic population, we sought to determine whether the pattern of maternal gestational weight gain was associated with clinically significant changes in the neonatal birth weight.

\section{Materials and Methods}

\section{Design and settings}

We performed a single-centre, retrospective study of patients enrolled in the prenatal service and delivered at Wyckoff Heights Medical Centre in Brooklyn, New York. The hospital is located in a community comprised of primarily Latinos living below the poverty line [8]. We enrolled women who received prenatal care and delivered a live born singleton gestation presenting to the obstetrics and gynaecology department between January $1^{\text {st }}$ to December 31 $31^{\text {st }}, 2017$ and delivered a singleton infant. This study was approved by our Institutional Review Board at Wyckoff Heights Medical Centre.

\section{Study population}

We enrolled a sample of adult women, 18 years or older, who received prenatal care and delivered at Wyckoff Heights Medical Centre. One thousand three hundred fifty-six charts were reviewed and eight hundred forty-five women met the inclusion criteria. Fourteen women who were classified as underweight at the initial prenatal visit were excluded due to inadequate sample size.

\section{Data collection and data management}

Baseline demographic data were collected on all enrolled women and neonates (Table 1). Maternal data such as maternal age at delivery, height, body weight at: first prenatal visit, 16-20 weeks' gestation, 24-28 weeks' gestation, 36-38 weeks' gestation, as well as reproductive characteristics such as gravidity, parity, mode of delivery, past medical history and blood which were extracted from the hospital medical record. Neonatal data including gestational age at delivery, birth weight, and neonatal intensive care unit admission (Figure 2) as well as length of stay were collected from the hospital medical record.

Table 1: Maternal and neonatal baseline characteristics.

\begin{tabular}{|c|c|c|c|}
\hline & \multicolumn{3}{|c|}{ Maternal BMI } \\
\hline & Normal weight & Over weight & Obese \\
\hline Average maternal age (years) & 26.7 & 28.5 & 29 \\
\hline \multicolumn{4}{|c|}{ Maternal ethnicity (\%) } \\
\hline Caucasian & 3.4 & 2 & 0.0 \\
\hline African American & 10.2 & 11.0 & 15.5 \\
\hline Hispanic & 72.8 & 73.5 & 70.0 \\
\hline Asian & 1.5 & 1.2 & 0.0 \\
\hline Others & 2.1 & 12.3 & 14.5 \\
\hline Height $(\mathrm{cm})$ & 63 & 63.1 & 63 \\
\hline Weight (kg) & 126.3 & 154.3 & 202.9 \\
\hline BMI $(\mathrm{kg} / \mathrm{m} 2)$ & 22.4 & 27.2 & 35.9 \\
\hline GA at initial BMI check (weeks) & 12.1 & 4.9 & 16 \\
\hline \multicolumn{4}{|l|}{ Gravidity (n) } \\
\hline 1 & 89 & 63.0 & 62.0 \\
\hline$>2$ & 178 & 201 & 233 \\
\hline Average birth weight (g) & 3230.9 & 3340.6 & 3377.3 \\
\hline NICU admission (\%) & 13 & 10 & 15 \\
\hline
\end{tabular}

Gestational age was calculated from the first day of the last menstrual period which was confirmed by ultrasound, or from the first dating ultrasound scan performed if the last menstrual period was unknown [9].

We conducted analyses of birth weight based on gestational age. Large for gestational age (LGA) and small for gestational age (SGA) were based on the $90^{\text {th }}$ and $10^{\text {th }}$ percentiles of weight for gestational age, respectively, based on the Duryea birth weight chart [10].

\section{Measurements}

Bodymassindex was calculated as the woman's firstdocumented pregnancy weight in kilograms divided by height in squared meters. Based on BMI, women were classified as normal weight (18.5-24.9 
$\mathrm{kg} / \mathrm{m}^{2}$ ), overweight (25.0 to $29.9 \mathrm{~kg} / \mathrm{m}^{2}$ ) or obese ( $\geq 30 \mathrm{~kg} / \mathrm{m}^{2}$ ). According to the 2009 IOM GWG recommendations, patients who are normal weight should gain $25-35 \mathrm{lbs}$ with an average weight gain of $1 \mathrm{lb}$ per week in the second and third trimester [11]. Patients categorized as overweight, should gain 15-25lbs with an average weight gain of $0.6 \mathrm{lbs}$ per week in the second and third trimester, and lastly patients categorized as obese, should gain 11-20lbs with an average weight gain of $0.5 \mathrm{lbs}$ per week in the second and third trimester [11].

Gestational weight gain was calculated by subtracting the pregnancy weight measured at the first prenatal visit from the weight recorded at the 36-38-week visit. This gestational weight gain was then compared with the IOM recommended GWG for each BMI category and categorized as inadequate, adequate or excessive. Specifically, adequate GWG was defined as weight gain of 25 to 35 pounds for women of normal BMI, 15 to 25 pounds was for overweight women and 11 to 20 pounds for obese women. Inadequate and excessive GWG were defined as less and more than adequate GWG according to the IOM guidelines, respectively. Gestational weight gain and body mass index were also examined with respect to other sociodemographic and health determinants. Ethnicity was self-reported by different ethnic categories such as: Caucasian, Hispanic, Asian, African American, or Other.

\section{Statistical analysis}

Statistical analyses were performed using STATA software package (STATA version 15.1, College Station, Texas). We used Wald chi-square tests to identify statistically significant differences of women gaining inadequate, adequate or excessive weight in each category. We examined associations between characteristics of interest and gestational weight gain adequacy using multinomial logistic regression to estimate odds ratios (OR) and 95\% confidence intervals (CI). Statistical significance was claimed at $\mathrm{P}<0.05$.

\section{Results and Discussion}

\section{Missing data}

In order to explore potential sample bias based on missing data, the patients who were excluded from the analyses due to missing first visit pregnancy weight, height, or gestational weight gain data were compared to those without missing data on these variables with respect to sociodemographic and health parameters (Table 1).

\section{Maternal characteristics}

The majority of the mothers in the study population, $71.92 \%$, were of Hispanic origin, 12.01\% were African American, 3.25\% were of Caucasian origin and $12.82 \%$ were from other origins.

The prevalence of multiparous women in the normal weight, overweight and obese categories were $66.67 \%, 76.14 \%$, and $78.98 \%$ respectively. However, the prevalence of primiparous women in the normal weight, overweight and obese categories were $33.33 \%, 23.86 \%, 21.02 \%$, respectively. We also observed that the obese population were more likely to commence prenatal care later than that seen for the underweight, normal weight and overweight population. (Table 1).

\section{Gestational weight gain by Body Mass Index}

The prevalence of normal weight, overweight and obese women was 31.83\%, 31.12\%, 35.38\% respectively. By different categories, the prevalence of adequate GWG, by different groups was: normal weight, $27.9 \%$, overweight, $34.5 \%$, and obese, $24.5 \%$. The prevalence of inadequate GWG by different groups were: $48.9 \%, 31.0 \%, 40.6 \%$ for normal weight, overweight and obese mothers respectively. Lastly, the prevalence of excessive GWG by different groups was: $23.1 \%, 35.8 \%$, and $37.5 \%$ for normal weight, overweight and obese mothers respectively (Figure 1).

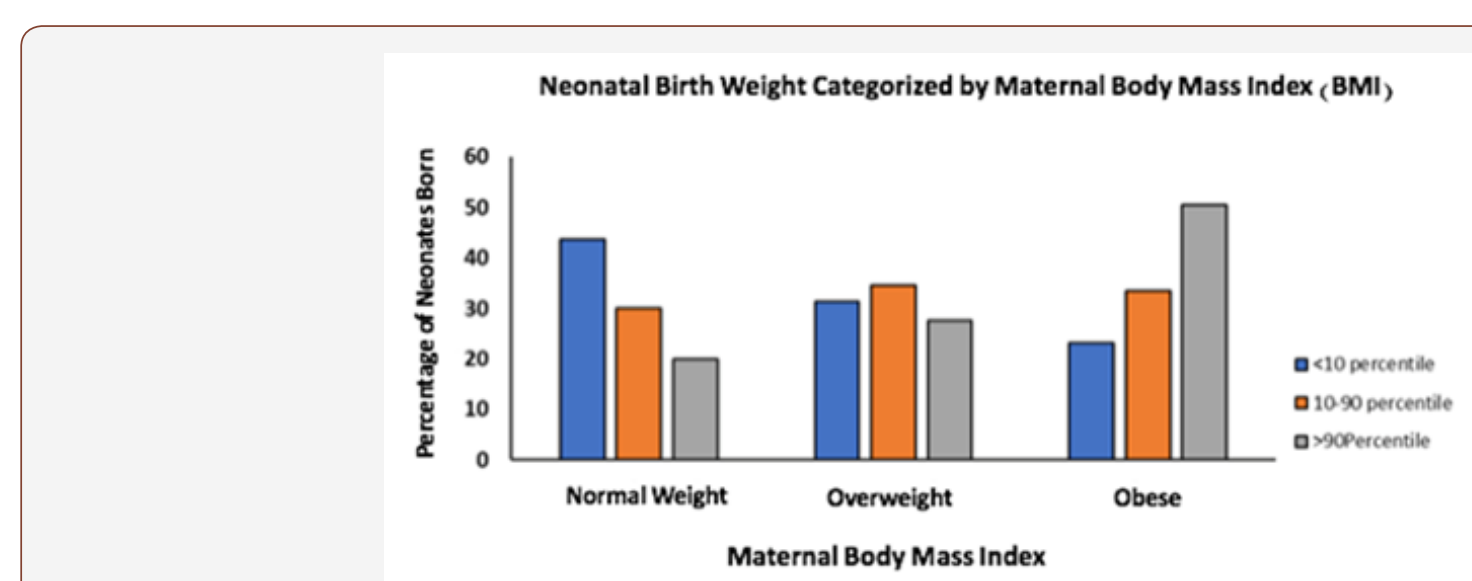

Figure 1: Duryea birth weight percentage of neonates born based on maternal BMI.

Wyckoff Heights Medical Centre, NY, 2017

We also calculated the prevalence of GWG in each trimester according to the woman's BMI. In the first trimester women gained $4.43 \pm 5.42 \mathrm{lb}, 2.28 \pm 15.03 \mathrm{lb}, 1.96 \pm 8.07 \mathrm{lb}$ (mean $\pm \mathrm{SEM}$ ), for the normal weight, overweight or obese populations respectively. In the second trimester, the prevalence of gestational weight gained was $18.41 \pm 34.68 \mathrm{lb}, 11.73 \pm 4.31 \mathrm{lb}, 9.34 \pm 5.56 \mathrm{lb}$ (mean \pm SEM), for the normal weight, overweight and obese populations respectively. Lastly, in the third trimester the GWG was $26.47 \pm 5.70 \mathrm{lb}$, $20.78 \pm 6.33 \mathrm{lb}, 16.81 \pm 6.83 \mathrm{lb}$ (mean \pm SEM); for the normal weight, overweight and obese populations respectively. 
The odds of excessive weight gain were 1.85 times higher in the obese population compared to the normal weight and overweight population (OR=1.85 95\% CI 1.10-3.14; p>0.05) (Table 2).

\section{Neonatal birth weight categorized by maternal Body Mass Index}

The odds of having a LGA ( $>90$ percentile) infant were 2.33 times higher in obese than normal BMI women (OR=2.33 95\% CI
1.15-4.97; $p>0.05)$. Conversely, the odds of having a SGA infant were 0.47 times lower in obese than normal BMI women $\mathrm{OR}=0.47,95 \%$ CI 0.24-0.92; $\mathrm{p}>0.05$ ). However, the odds of having a LGA infant were higher in those with excessive GWG and lower in those with inadequate GWG compared to those with adequate GWG (Table 3). No significant association was found between overweight mothers with SGA or LGA infants, (OR=0.62, 95\% CI 0.33-1.14; p>0.05) (OR=1.19, 95\% CI 0.53-2.74; $\mathrm{p}>0.05$ ), respectively.

Table 2: Adjusted Odds Ratios (OR) and 95\% Confidence Interval for association between maternal Body Mass Index (BMI) and Gestational Weight Gain (GWG).

\begin{tabular}{|c|c|c|c|}
\hline \multicolumn{2}{|c|}{ Total (n), \% } & Inadequate GWG OR $(95 \% \mathrm{Cl})$ & Excessive GWG OR $(95 \% \mathrm{Cl})$ \\
\hline \multicolumn{4}{|c|}{ Mother BMI } \\
\hline Normal weight & $268,31.8$ & Referent & Referent \\
\hline Over weight & $263,31.3$ & $0.54(0.32,0.82)$ & $1.25(0.76,2.08)$ \\
\hline Obese & $299,35.4$ & $0.95(0.59,1.53)$ & $1.85(1.10,3.14)$ \\
\hline
\end{tabular}

Bolded value represents statically significant associations

Table 3: Neonatal birth weight outcome among term infants compared to maternal Body Mass Index (BMI).

\begin{tabular}{|c|c|c|c|c|c|c|}
\hline \multirow{2}{*}{ Duryea Birth (g) } & \multirow{2}{*}{ Percentile } & \multirow{2}{*}{$\begin{array}{c}\text { Gestational Age } \\
\text { (Weeks) }\end{array}$} & \multirow{2}{*}{$\%$} & \multicolumn{3}{|c|}{ Neonatal Birth weight by Maternal BMI } \\
\hline & & & & $18.5-24.9$ & $24.9-30$ & $>30$ \\
\hline \multirow{2}{*}{2500} & $10^{\text {th }}$ & 37 & 0.3 & 2147.0 & $\mathrm{n} / \mathrm{a}$ & $\mathrm{n} / \mathrm{a}$ \\
\hline & $11-89^{\text {th }}$ & 37 & 4.3 & 2929.6 & 3065.6 & 3093.5 \\
\hline 3612 & $90^{\text {th }}$ & 37 & 1.1 & 3786.5 & $\mathrm{n} / \mathrm{a}$ & 3789.7 \\
\hline \multirow{2}{*}{2706} & $10^{\text {th }}$ & 38 & 2.2 & 2516.2 & 2535.7 & 2310.0 \\
\hline & $11-89^{\text {th }}$ & 38 & 11.4 & 3247.8 & 3222.3 & 3231.7 \\
\hline 3799 & $90^{\text {th }}$ & 38 & 1.7 & 3820.0 & 4129.7 & 4048.2 \\
\hline \multirow{2}{*}{2877} & $10^{\text {th }}$ & 39 & 3.9 & 2708.5 & 2782.2 & 2729.3 \\
\hline & $11-89^{\text {th }}$ & 39 & 34 & 3269.8 & 3387 & 3385.5 \\
\hline 3941 & $90^{\text {th }}$ & 39 & 4.3 & 4160.7 & 4390.6 & 4318.4 \\
\hline \multirow{2}{*}{3005} & $10^{\text {th }}$ & 40 & 2.5 & 2853.4 & 2788.2 & 2705.8 \\
\hline & $11-89^{\text {th }}$ & 40 & 23.4 & 3487.9 & 3613.1 & 3567.9 \\
\hline 4057 & $90^{\text {th }}$ & 40 & 1.7 & 4455.7 & 4430.0 & 4254.2 \\
\hline
\end{tabular}

\section{Neonatal ICU Admission Categorized by Maternal Body Mass Index}

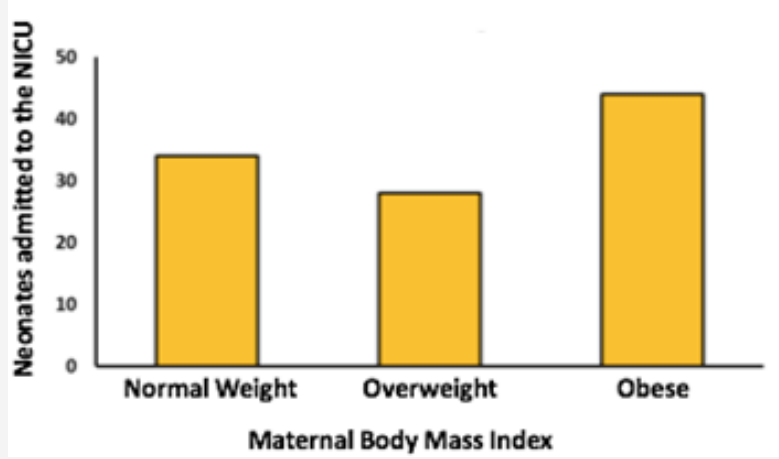

Figure 2: Number of neonates admitted to the Neonatal Intensive care unit by maternal BMI. Wyckoff Heights Medical Centre, NY, 2017

A key finding in our study was that the obese population had a higher prevalence of NICU admission compared to the underweight, normal weight or overweight populations (Figure 2).

\section{Discussion}

Nationally, it has been reported that over seventy percent of Hispanic women fall into the overweight and/or obese category [1]. 
Among Hispanic women, between thirty-six and fifty percent have excessive gestational weight gain and between seventeen to thirty percent have inadequate gestational weight gain depending on the national origin of the patient [1]. Both inadequate and excessive gestational weight gain have been shown to have detrimental effects on both the health of the woman and the developing fetus including: preterm birth, small for gestational age (SGA) neonates, large for gestational age (LGA) neonates, prolonged labour, shoulder dystocia, postpartum haemorrhage and delivery via caesarean section [12-17].

In our study, over $35 \%$ of the overweight and obese populations were found to have excessive weight gain. Similar to our study, Chasen-Taber et al. studied a predominantly Puerto Rican population and found that almost $50 \%$ of the women gained above the recommended weight gain set forth by the IOM $[6,17]$. Our study also determined that the possibility of having a large for gestational age neonate ( $>90$ percentile) were 2.33 times higher in the obese population compared to the normal weight population $[13,17]$. Another study demonstrated the rate of macrosomia, birth weight $>4000 \mathrm{~g}$, to be highest among women with excessive weight gain $[1,17]$.

Excessive gestational weight gain has also been correlated with an increased number of neonatal intensive care unit (NICU) admissions, which our study demonstrated (Figure 2). It has been shown that neonates born to morbidly obese women have a doubled risk of birth injuries and respiratory distress syndrome, a threefold increased risk of bacterial sepsis, birth asphyxia, and feeding problems, and a fourfold increased risk of birth injuries to the peripheral nervous system and hypoglycaemia, regardless of mode of delivery [12,15]. Furthermore, these neonates are more likely to have low 5 minute Apgar scores, seizures, hypoglycaemia, polycythaemia, and meconium aspiration syndrome [12].

Not only does overall excessive gestational weight gain lead to a plethora of maternal and neonatal problems, some studies have shown that trimester specific weight gain can be of more importance. Our study showed that during the $3^{\text {rd }}$ trimester, women gained the most amount of weight, in pounds, for all BMI groups: $26.47 \pm 5.70 \mathrm{lbs}, 20.78 \pm 6.33 \mathrm{lbs}, 16.81 \pm 6.83 \mathrm{lbs}$ (mean $\pm \mathrm{SEM}$ ); for the normal weight, overweight and obese populations respectively. Siega-Riz AM, et al. [19] also studied a group of Hispanic women in California and found that the mean total weight gain for each category in the third trimester was $28.010 .21 \mathrm{lbs}, 24.3 \pm 0.46 \mathrm{lbs}$, and $22.2 \pm 0.52 \mathrm{lbs}$ for normal weight, overweight, and obese women, respectively. Similar to our study, the women in the normal weight group gained more weight compared to the women in the overweight and obese groups. In a study conducted by Siega-Riz $\mathrm{AM}$, et al, [20] they found that in the third trimester, women who delivered term neonates gained on average, $30 \mathrm{~g}$ more per week than women who delivered preterm neonates. To the contrary, Kaminarek et al. found that inadequate weight gain in the third trimester increased the risk of preterm birth by $90 \%$, as well as
Ryan et al, who demonstrated that inadequate weight gain after 20 weeks of gestation was associated with preterm birth [12,21]. However, our study did not demonstrate any significant association between inadequate weight gain and overall preterm birth.

It has been shown that pre-pregnancy weight is directly associated with gestational weight gain. This vital sign can be of utmost importance for obstetricians and gynaecologists in guiding management of a future pregnancy. A study conducted by SangiHaghpeykar H, et. al [1] found that 59\% of Hispanic women were overweight or obese prior to conception and almost half of the women had excessive gestational weight gain. Another study by Chasen, et.al [22] determined that $44 \%$ of women were classified as overweight or obese pre-pregnancy. In our study, we found that over $30 \%$ of the women were noted to be overweight or obese at their initial prenatal appointment, at which point it can be very difficult to control one's weight. A high pre-pregnancy BMI can be detrimental to a woman's pregnancy due to the comorbidities that occur with obesity, namely diabetes, hypertension, hypercholesterolemia, and metabolic syndrome [12,17].

Our study showed that obese women were more likely to commence prenatal care later than that seen for the underweight, normal weight and overweight populations. Establishing prenatal care early with the ability to address body mass index, nutritional requirements, diet, exercise, etc. is very important to the growth and development of the fetus as well as the health of the mother. It has been shown that women who start prenatal care later are at increased risk of neonatal complications such as; congenital abnormalities, prematurity and fetal death as well as maternal complications with increased rates of maternal morbidity and mortality [21]. Often times women at high risk for pregnancy complications, especially those with high pre-pregnancy BMI are screened early in pregnancy for diseases such as hypertension, diabetes, and preeclampsia. Consistent with other studies of Hispanic women, our study demonstrated that inadequate prenatal care is a risk factor for insufficient weight gain.

Although our study was limited by not having the pre-pregnancy weight on our patients, most of them had initiated prenatal care during the first trimester. Another limitation was that our results may not be generalizable to all Hispanic women since our patients came from different South and Central American, as well as Caribbean countries and included second-generation immigrants.

\section{Conclusions}

Maternal weight gain monitoring is a very valuable clinical tool in assessing the health of pregnant women. Despite the Institute of Medicine's guidelines for weight gain in pregnancy, many women, exceed the recommendations putting their health as well as the growth and well-being of their fetus at risk.

The impact of excessive gestational weight gain in this predominantly Hispanic population is the added burden of maternal 
and neonatal complications on a background of suboptimal outcome due to associated comorbidities. Socio-cultural as well as economic factors may negatively influence the nutritional profile of these families and hence their pre-pregnancy and prenatal health status. Improving pregnancy outcome for mothers and new-borns will include measures to introduce to these women daily health promoting habits well before conception and continued during the pregnancy with consistent monitoring and positive reinforcement.

Our study showed that obese women are more likely to have a large for gestational age neonate who can increase neonatal morbidity and mortality. More specifically, pre-pregnancy and prenatal counselling should stress the nutritional requirements during pregnancy, information about diet and exercise, availability of weight loss programs prior to pregnancy including consultation with a nutritionist, and possible pharmacotherapy. In addition, women should be encouraged to monitor their weight gain during pregnancy, especially during the first half of the process to ensure the early adoption of good nutritional habits and improved maternal compliance during the remainder of their gestation.

\section{Key Messages}

In our predominantly mixed Hispanic patient population, our study showed a significant association between excessive gestational weight gain and large for gestational age at birth. Dietary intervention before and possibly during the 1st trimester can potentially reduce, if not eliminate this complication.

\section{Acknowledgement}

None.

\section{Conflict of Interest}

Authors declare no conflict of interest.

\section{References}

1. Sangi-Haghpeykar H, Lam K, Raine SP (2014) Gestational weight gain among Hispanic women. Matern Child Health J 18(1): 153-160.

2. Savitz DA, Stein CR, Siega-Riz AM, Herring AH (2011) Gestational weight gain and birth outcome in relation to prepregnancy body mass index and ethnicity. Ann epidemiol 21(2): 78-85.

3. Walker LO, Minseong K (2002) Psychosocial thriving during late pregnancy: relationship to ethnicity, gestational weight gain, and birth weight. J Obstet Gynecol Neonatal Nurs 31(3): 263-274.

4. Kramer MS (1998) Socioeconomic determinants of intrauterine growth retardation. Eur J Clin Nutr 52 Suppl 1: S29-S32.

5. (2009) Institute of Medicine. Weight gain during pregnancy: reexamining the guidelines. Washington, DC: National Academies Press, USA.
6. Chasan-Taber L, Silveira M, Lynch KE, Pekow P, Solomon CG, et al. (2014) Physical activity and gestational weight gain in Hispanic women. Obesity 22(3): 909-918.

7. Cheney K, Berkemeier S, Sim KA, Gordon A, Black A (2017) Prevalence and predictors of early gestational weight gain associated with obesity risk in a diverse Australian antenatal population: a cross-sectional study. BMC Pregnancy Childbirth 17(1): 296.

8. (2011) Bushwick Demographics. The food communities of NYC.

9. American College of Obstetricians and Gynecologists (2017) ACOG Committee opinion no. 700: Methods for Estimating the Due Date. Obstet Gynecol 129(5): e150-e154.

10. Duryea EL, Hawkins JS, McIntire DD, Casey BM, Leveno KJ (2014) A Revised Birth Weight Reference for the United States. Obstet Gynecol 124(1): 16-22.

11. American College of Obstetricians and Gynecologists (2013) ACOG Committee opinion no. 548: weight gain during pregnancy. Obstet Gynecol 121(1): 210-212.

12. Kominiarek MA, Peaceman AM (2017) Gestational weight gain. Am J Obstet Gynecol 217(6 ): 642-651.

13. Blomberg M (2013) Maternal obesity, mode of delivery, and neonatal outcome. Obstet Gynecol 122(1): 50-55.

14. Blomberg M (2011) Maternal and neonatal outcomes among obese women with weight gain below the new Institute of Medicine recommendations. Obstet Gynecol 117(5): 1065-1070.

15. Stotland NE Cheng YW, Hopkins LM, Caughey AB (2006) Gestational weight gain and adverse neonatal outcome among term infants. Obstet Gynecol 108(3): 635-643.

16. Crane JM, White J, Murphy P, Burrage L, Hutchens, et al. (2009) The effect of gestational weight gain by body mass index on maternal and neonatal outcomes. J Obstet Gynaecol Can 31(1): 28-35.

17. Cheng YW, Chung JH, Kurbisch-Block I, Inturrisi M, Shafer S, et al. (2008) Gestational weight gain and gestational diabetes mellitus: perinatal outcomes. Obstet Gynecol 112(5): 1015-1022.

18. Ludwig DS, Currie J (2010) The association between pregnancy weight gain and birthweight: a within-family comparison. The Lancet 376(9745): 984-990.

19. Siega-Riz AM, Hobel CJ (1997) Predictors of poor maternal weight gain from baseline anthropometric, psychpsocial, and demographic information in a Hispanic population. J Am Diet Assoc 97(11): 12641268.

20. Siega-Riz AM, Adair LS, Hobel CJ (1996) Maternal underweight status and inadequate rate of weight gain during the third trimester of pregnancy increases the risk of preterm delivery. The J nutr 126(1): 146-153.

21. Ryan Jr GM, Sweeney PJ, Solola AS (1980) Prenatal care and pregnancy outcome. American Journal of Obstetrics and Gynecology 137(8): 876881.

22. Chasan-Taber L, Schmidt MD, Pekow P, Sternfeld B, Solomon CG, et al., Predictors of excessive and inadequate gestational weight gain in Hispanic women. Obesity16(7): 1657-1666. 